\title{
Dinâmica regional e setorial do emprego no Brasil: 1997 a 2007
}

José Antonio Rodrigues da Silva ${ }^{1}$

Paulo Aguiar do Monte $^{2}$

Resumo: A evolução do mercado de trabalho é considerada um dos fatores críticos no processo de desenvolvimento econômico, e o seu crescimento ocorre de forma mais rápida em algumas regiões ou setores do que em outros. O objetivo deste trabalho é examinar a dinâmica regional do emprego no Brasil, no período 1997-2007, utilizando a análise shift-share na versão alternativa de Esteban-Marquillas (1972). Esse modelo permite a decomposição da variação do emprego em quatro componentes ou efeitos: nacional, estrutural, regional e alocação. Neste estudo são utilizados os dados da Relação Anual de Informações Sociais - RAIS, fornecidos pelo Ministério do Trabalho e Emprego - MTE, desagregados em grandes setores da economia (inicialmente 8 setores e, posteriormente, em 25 setores). O principal resultado obtido no estudo é que, apesar do incremento no volume de empregos gerados no Brasil e em todas as regiões, durante os anos analisados, o efeito estrutural tem uma influência mínima na explicação do crescimento do emprego nas regiões.

Palavras-chave: Desenvolvimento regional; Emprego; Shift-share.

1 Professor do Departamento do Departamento de Relações Internacionais da UFPB. E-mail: jarsilva@uol. com.br

2 Professor do Departamento de Economia da UFPB. E-mail: pauloaguiardomonte@gmail.com 


\title{
Dynamic regional and sectoral employment in Brazil: 1997 to 2007
}

\begin{abstract}
The evolution of labour market is regarded as one of the critical factors in the process of economic development, and its growth occurs faster in some regions than others. The aim of this paper is to examine the regional dynamism of Brazilian employment change during the period 1997-2007 with the help of the traditional shift-share analysis an alternate version of the model EstebanMarquillas (1972). This model enables the decomposition of employment change into four components or effects: national share, industrial mix, competitive and allocation. The data employed in this study are the ones from the Annual Social Information - RAIS, provided by the Ministry of Labor and Employment - MTE, broken down into major economic sectors (at 8-sectors level of disaggregation initially and thereafter at an 25-sectors level of disaggregation). The main result found in this study is that only a minimal variation in regional employment growth is explained by the sectoral-mix effect.
\end{abstract}

Keywords: regional employment; employment; shift-Share.

\section{Introdução}

O emprego é uma das principais variáveis econômicas, cuja evolução ocorre de forma mais rápida em alguns setores do que em outros e, mais intensamente, em algumas regiões que em outras. Dado que a composição setorial do emprego varia entre as regiões, uma relação causal poderia ser esperada. Assim, não é de se estranhar que as disparidades regionais nas taxas de crescimento do emprego sejam frequentemente substanciais, especialmente em países de dimensões continentais.

Certamente, este tem sido o caso do Brasil, cuja economia vem passando por uma série de transformações com importantes desdobramentos sobre o mercado de trabalho nos últimos anos. Em decorrência da aceleração do processo de abertura comercial durante os anos 1990, da adoção de novas tecnologias de produção e da implantação de um amplo programa de estabilização, a economia brasileira iniciou uma nova dinâmica espacial, na qual algumas regiões experimentaram elevadas taxas de crescimento do emprego, enquanto outras têm registrado taxas reduzidas, ou mesmo negativas em alguns setores.

Até a década de sessenta, antes do Milagre Econômico, o desenvolvimento econômico brasileiro era concentrado em alguns estados e regiões, o que gerou grande concentração da renda e da produção. No auge da concentração, no inicio dos anos 1970, os Estados de São Paulo e Rio de Janeiro que, juntos, de- 
tinham menos de 30\% da população brasileira, e eram responsáveis por mais de $56 \%$ da produção nacional. Somente a partir desse período esta situação, lentamente porém de forma gradual, começou a se reverter, com a aceleração do processo de desconcentração e mudança espacial da dinâmica do emprego. (Pimentel \& Haddada 2005; Diniz \& Crocco, 1996 Barros et al. 2008).

Fatores como a abertura econômica e o baixo crescimento econômico impactaram, de forma mais intensa, as grandes cidades concentradoras da produção nacional, que, somados à guerra fiscal ocorrida entre os estados, foram fatores essenciais neste processo de realocação produtiva espacial. Azevedo e Toneto Júnior (2001) destacam que a realocação privada dos investimentos em busca, principalmente, de menores custos de produção, foi o principal fator da nova organização da produção industrial que ganhou intensidade com a abertura comercial.

Além da importância do entendimento do motivo porque o crescimento do emprego varia entre as regiões, cabe destacar que o incentivo à desconcentração regional e ao crescimento equilibrado e homogêneo deve ter um papel fundamental na definição de políticas regionais adequadas e eficientes.

Nesse contexto, este trabalho tem como objetivo apresentar uma visão geral sobre a dinâmica setorial-regional da evolução do emprego entre as regiões brasileiras no período 1997-2007, bem como para os dois qüinquênios distintos dessa década (1997-2002 e 2002-2007), por meio da utilização da analise shift-share, incorporando a introdução do conceito de emprego homotético. A divisão da década em duas etapas distintas é interessante não somente pelas características do modelo utilizado, como pelo fato de no período estudado ter sido observada uma transformação sensível nas condições internacionais. A primeira etapa, 1997-2002, foi caracterizada por um ambiente econômico externo bastante desfavorável, devido ao acentuado declínio no crescimento do comércio mundial, quando comparado com a década anterior (Cheptea et al. 2005). A segunda, 2002-2007, marca não somente o início de uma melhora nas condições externas, como também representa o começo de uma nova fase na condução da política econômica interna pela ascensão de um governo ligado às classes trabalhadoras ao poder central da nação, o qual adota uma série de medidas visando a aceleração da criação de empregos no país. Apesar da simplicidade e limitações atribuídas ao instrumento de análise aplicado neste estudo, seu valor como importante forma de abordagem para um exame inicial dos fatores explicativos da variação diferencial do crescimento entre as regiões tem sido reconhecido na literatura através do amplo uso. Os dados utilizados no estudo têm como fonte básica as estatísticas da Relação Anual de Informações Sociais (RAIS), disponibilizadas pelo Ministério do Trabalho e Emprego (MTE), para os anos 1997, 2002 e 2007, em nível macrorregional, subdivida conforme seus setores de atividades.

No sentido de atingir o objetivo acima especificado, o trabalho encontra-se estruturado como segue. Após esta introdução, na seção 2 apresenta-se uma 
breve contextualização da dinâmica do emprego no país.

Na seção 3, após uma breve análise do modelo shift-share tradicional e suas limitações, apresenta-se um modelo estendido contendo uma importante reformulação que possibilita a decomposição da variação do emprego em quatro efeitos (nacional, setorial, competitivo e alocação). Na seção seguinte são discutidos os principais resultados obtidos, e na parte final do trabalho são apresentadas as conclusões, seguidas das referências bibliográficas.

\section{Breve contextualização da dinâmica recente do empre- go no Brasil}

Com o processo de globalização, mais intenso a partir dos anos oitenta, mudanças significativas ocorreram na economia nacional, principalmente aquelas que visavam adaptar o processo de trabalho das empresas à nova realidade de um mercado cada vez mais competitivo. Nesse novo cenário de rápidas transformações econômicas, as mudanças ocorridas no ambiente internacional, sejam elas favoráveis ou não, impactaram de forma mais intensa a dinâmica do emprego nacional.

A economia brasileira nos anos oitenta foi marcada por um ambiente bastante desfavorável à expansão do emprego formal. Taxas elevadíssimas de inflação (chegando a 1.600\%, em 1986), ampliação das relações informais de trabalho e da precarização do trabalho, queda dos rendimentos, menores possibilidades de mobilidade social, concentração de renda e aumento da taxa de desemprego são algumas das características marcantes dessa década. (Montal 1998; Singer 1998). Para Cano (2008) os acontecimentos deste período acarretaram uma desconcentração por efeito estatístico (resultado da crise e seus impactos) e não decorrentes de expressivos aumentos territoriais de produção, visto que tanto São Paulo (o principal estado da Federação em termos de participação no Produto Interno Bruto) quanto o restante do Brasil apresentaram crescimento negativo em muitos ramos e segmentos industriais.

A partir dos anos noventa, com a estabilização da inflação e a criação de novas políticas públicas de emprego e renda - em especial, a criação do Fundo de Amparo ao Trabalhador e os Programas de Geração de Emprego e Renda, a dinâmica do mercado de trabalho começa a sofrer alteração. No entanto, as crises econômicas do período, tanto no plano nacional, como internacional, comprometeram o crescimento do PIB; e, consequentemente, a expansão do emprego formal no país, bem como a redução das taxas de desemprego e a sua duração. Na década de noventa a elevação do produto nacional foi inferior a $2,5 \%$ ao ano.

Segundo Barros et al. (2008), as transformações sofridas pelo mercado de trabalho 
durante a década passada resultaram de um cenário macroeconômico conturbado, de mudanças na condução das políticas públicas e de avanços tecnológicos, que culminou na redução do emprego formal e expansão das relações informais de trabalho. ${ }^{3}$ Ambrózio (2009) afirma que, entre 1995 e meados de 1999, o nível de ocupação permaneceu relativamente estagnado, com a taxa de desemprego alcançando um patamar muito elevado nos anos de 1998 e 1999, em consequência de choques externos adversos derivados das crises da Ásia e da Rússia, só vindo a se restabelecer a partir do segundo semestre de 1999, quando houve substancial aumento no nível de ocupação.

Somente a partir da virada do século, mais especificamente a partir de 2002, é que o ambiente econômico começou a se alterar positivamente. O Brasil passou a apresentar taxas elevadas de crescimento econômico e expansão significativa no volume de empregos gerados impulsionado pelo cenário de crescimento econômico mundial. Segundo dados do Instituto Brasileiro de Geografia e Estatística - IBGE, o Produto Interno Bruto brasileiro - PIB, cresceu a uma taxa superior a 4,5\% entre 2004 e 2007. Ao mesmo tempo, a expansão no mercado de trabalho formal no período 2000 e 2007 ultrapassou a taxa de 5,0\% ao ano. O número de trabalhadores formalmente empregados saltou de 27 milhões para cerca de 36 milhões.

Neste novo contexto de expansão da atividade econômica, algumas regiões têm se destacado, em especial as menos favorecidas economicamente. Prova disto é que, desde 2001, a economia nordestina vem crescendo a taxas superiores à média nacional (4,2\% ao ano contra 2,3\% do Brasil, segundo o IBGE). Esses resultados recentes da economia brasileira, em especial, podem ser traduzidos também em números de empregos gerados no mercado de trabalho. No Nordeste, entre 2000 e 2007, houve um aumento de 50\% no número de assalariados com contratos de trabalho, saltando de 4.374.850 para 6.567.837 o número de trabalhadores nessa condição, o que representa um aumento de cerca de $8 \%$ ao ano; enquanto no Norte, neste mesmo período, o número de trabalhadores no mercado formal aumentou quase $80 \%$, saindo de 1.094.365 para 1.954.641, em 2007. Esta retomada do crescimento econômico brasileiro foi fundamental para a redução da taxa de desemprego que caiu de 12,3\% (2003) para 8,5\% (2007); ou seja, uma queda de 3,8 pontos percentuais. Um variável importante nessa mudança de cenário diz respeito a Guerra Fiscal adotada no país que contribuiu para dispersar as atividades nos estados. Segundo Cano (2008) a Guerra Fiscal alterou, embora artificialmente, a localização de pequena fração da produção industrial, fazendo com que aumentasse essa desconcentração aparente.

Além das diferenças regionais destacadas, este cenário de crescimento eco-

3 Segundo Tafner (2006), o aumento da informalidade no Brasil, durante a década de noventa, ocorreu tanto em âmbito setorial quanto espacial, e, principalmente, nas áreas metropolitanas. 
nômico e melhoria dos indicadores no mercado de trabalho ocorreu de forma heterogênea, também, do ponto de vista setorial. A mudança no padrão recente de geração de emprego foi particularmente intensa quando se considera a indústria de transformação. Ramos e Ferreira (2005) analisaram o comportamento heterogêneo do emprego na indústria no período 1992/2002 e observaram que houve uma destruição líquida significativa de postos de trabalho nas áreas metropolitanas (mais de 300 mil) e uma criação líquida nas áreas não metropolitanas (mais de 1 milhão). Ambrózio (2009) destaca que, entre 1996 e 1999, houve nesse setor uma destruição líquida de cerca de 300 mil postos de trabalho (50 mil por ano), enquanto no período 2000-2005, houve uma recuperação de modo expressivo, quando foram criados, liquidamente, cerca de 1,5 milhões de novos postos na indústria de transformação (300 mil por ano). Como resultado, esse setor perdeu participação no emprego total, entre 1996 e 1999 (caindo de 20,8\% para 18,4\%) e, depois, manteve estável a sua participação, apesar do crescimento observado, em 18,5\% em 2005. Este resultado, todavia, contrasta com o setor de comércio, que apresentou expressivo aumento da sua participação.

Desta forma, o quadro apresentado a partir do início da década de 2000 é de uma realocação do emprego, tanto regional quanto setorial, para fora dos seus antigos grandes eixos e setores de geração de emprego, embora ainda lento, mas progressivo.

\section{Aspectos metodológicos e Base de dados}

\subsection{O modelo shift-share tradicional}

Como salientam Fernandez e Menédez (2002), a análise shift-share tem obtido um grande sucesso desde sua origem dentro da ciência regional devido a sua ampla variedade de possibilidades de aplicação. Sua popularidade reside fundamentalmente em três fatos: é simples de usar, fácil de entender e requer uma quantidade muito pequena de dados facilmente disponíveis. A despeito de sua larga utilização entre planejadores, geógrafos, economistas e cientistas regionais, essa técnica tem sido fortemente criticada.

Apesar da existência de alguns problemas na utilização do modelo shift-share, que serão discutidos a seguir, este serve como um importante instrumento de análise para separar a contribuição da composição industrial de uma região dos outros fatores locais de desenvolvimento. Como destaca Merrifield (1983), há um consenso geral que uma aplicação cuidadosa dessa técnica pode produzir conhecimentos que, apesar de modestos, possuem qualidade suficiente para explicar o desenvolvimento regional. 
O modelo shift-share tem sido usado na análise regional desde os anos $1940^{4}$, mas sua versão atual é atribuída a Dunn (1960). Na sua abordagem clássica, essa técnica tem como propósito decompor a evolução de uma magnitude econômica de uma região entre dois períodos de tempo em três fatores distintos: área de referência, estrutura industrial e deslocamento diferencial. A soma dos três componentes deve corresponder à variação real da variável decomposta, a qual pode ser a renda, o emprego, o produto, ou uma variedade de outras medidas (Dinc \& Haynes 2005).

O primeiro componente, também denominado de efeito ou participação nacional (para áreas menores, como municípios, por exemplo, pode ser utilizada a economia estadual como área de referência), constitui-se no valor básico para a determinação do crescimento esperado para a região e coincide com a taxa de crescimento calculada para o país como um todo. O segundo componente, também denominado de efeito setorial, mede a composição industrial da região e reflete o grau em que a área se especializa em indústrias que crescem a taxas maiores ou menores que as observadas em nível nacional. Então, se a região contém uma grande participação em indústrias que estão crescendo mais lentamente ou rapidamente em nível nacional, esta terá um deslocamento proporcional negativo ou positivo. O terceiro e último componente, usualmente denominado de efeito competitivo ou espacial, descreve a capacidade da economia local se expandir de forma mais acelerada ou mais lenta que as taxas de crescimento observadas nas indústrias da área de referência. A vantagem ou desvantagem comparativa observada pode depender de uma série de fatores, incluindo os efeitos de políticas governamentais, dotação natural da área, clima, ou outras, como a capacidade empresarial existente na região.

Em síntese, o modelo shift-share é um método descritivo e extremamente prático para avaliar os impactos de mudanças estruturais em economias locais ou regionais, a partir da observação de que é possível separar, estatisticamente e para qualquer intervalo de tempo, os componentes do crescimento dentro de uma região, que reflitam o crescimento nacional, o "mix" de atividades dentro da região e sua posição competitiva.

Algebricamente, o modelo shift-share pode ser definido como segue:

$$
E_{i j}^{t}-E_{i j}^{0}=\Delta E_{i j}=E_{i j} r_{n}+E_{i j}\left(r_{i n}-r_{n}\right)+E_{i j}\left(r_{i j}-r_{i n}\right)
$$

Onde, $E_{i j}=$ emprego na indústria ou setor $i$ na região $j ; r_{i j}=$ mudança

4 Na Inglaterra, sua origem pode ser traçada desde o famoso Barlow Report (1940) e, nos Estados Unidos podemos referenciar o trabalho de Creamer (1942, apud Selting \& Loveridge 1992). 
percentual no emprego no setor $i$ na região $j ; r_{i}=$ mudança percentual no emprego nacional no setor $i ; r_{n}=$ mudança percentual do emprego total em todos os setores na nação; $t=$ ano final do estudo; $\mathrm{o}=$ ano inicial do estudo.

Os três termos dessa identidade correspondem aos efeitos:

Efeito Nacional

Efeito Setorial ou Estrutural

Efeito Competitivo ou Regional

$$
E N_{i j}=E_{i j} r_{n}
$$

$$
E S_{i j}=E_{i j}\left(r_{i n}-r_{n}\right)
$$

Dado que, $r_{i j}, r_{i n}$ e $r_{n}$ são taxas de crescimento entre dois períodos de tempo, uma versão ampliada do modelo shift-share apresentado na equação 1 é:

$$
\begin{gathered}
\Delta E=E N_{i j}+E S_{i j}+E C_{i j}= \\
E_{i j}^{0}\left(\frac{E_{n}^{t}-E_{n}^{0}}{E_{n}^{0}}\right)+E_{i j}^{0}\left(\frac{E_{i n}^{t}-E_{i n}^{0}}{E_{i n}^{0}}-\frac{E_{n}^{t}-E_{n}^{0}}{E_{n}^{0}}\right)+E_{i j}^{0}\left(\frac{E_{i j}^{t}-E_{i j}^{0}}{E_{i j}^{0}}-\frac{E_{i n}^{t}-E_{i n}^{0}}{E_{i n}^{0}}\right)
\end{gathered}
$$

Onde, $i=$ número de setores ou indústrias em uma região ou nação; $j=$ número de regiões em uma área geográfica; $E=$ nível de emprego; $E_{i j}=$ emprego no setor $i$ na região $j ; E_{i n}=$ emprego nacional no setor $i=\Sigma_{j} E_{i j}$; $E_{n}=$ emprego total em todos os setores na nação $=\Sigma_{i} \Sigma_{j} E_{\dot{i j}}$

Em outras palavras, pode-se afirmar que a análise shift-share é uma técnica que permite decompor a variação emprego de uma região em duas análises distintas. A primeira, de participação (share analysis), ocupa-se com a parte proporcional do emprego nacional que é encontrada na região. A segunda, denominada de deslocamento (shift analysis), trata das mudanças regionais do emprego entre dois momentos de tempo, concentrando-se, em particular, em determinar se a mudança regional é maior ou menor do que a média nacional (isto é, se o deslocamento se faz para cima ou para baixo em relação à média nacional). Por sua vez, o deslocamento total do emprego (total shift) mede a diferença entre a mudança regional efetiva e a mudança que teria 
ocorrido se a região tivesse crescido às taxas nacionais. Ele se divide em dois componentes: o deslocamento proporcional ou estrutural; e o deslocamento diferencial ou regional.

Assim, as diferenças de crescimento que poderão ocorrer advindas desses dois componentes irão se refletir nas variações do crescimento real apresentado na região e as variáveis teóricas, o que deverá ocorrer caso o setor analisado da região não apresente as mesmas taxas de crescimento nacional.

Examinando os componentes acima, verificamos que o primeiro elemento, o efeito nacional, refere-se à participação da região na variação do emprego nacional. Quanto maior o crescimento do emprego no país como um todo, mais rápido deverá ser o aumento da oferta de trabalho na região. O segundo elemento é o componente estrutural, que corresponde à diferença entre a variação no emprego setorial que poderíamos esperar (dado sua estrutura industrial e as taxas de crescimento nacional de cada setor) e o efeito nacional. Assim, se a região possui uma estrutura industrial favorável é de se esperar que este componente seja positivo. Caso contrário, se a região fosse dotada de uma estrutura setorial desfavorável, poderíamos esperar um valor negativo. Finalmente, o terceiro elemento é o elemento residual, que mostra a diferença entre a mudança observada no emprego e a mudança que poderíamos ter esperado se todos os setores da região tivessem apresentado as mesmas taxas de crescimento observadas para o país. Este é simplesmente aquela parcela do crescimento regional que permanece inexplicada e para a qual pode ser dada uma série de interpretações. Um resíduo positivo significa que a taxa de crescimento da região excedeu a taxa de crescimento que poderia ter ocorrido se cada indústria da região tivesse crescido como sua contraparte nacional. Um resíduo negativo tem, naturalmente, o significado inverso.

Parece haver um consenso de que, embora não seja capaz de dar uma explicação precisa dos diferentes crescimentos regionais, a análise shift-share se constitui num método bastante útil para se fazer uma análise inicial desse crescimento. Pois, ao menos permite eliminar a possível influência de um vasto número de fatores sobre o crescimento de algumas regiões. O ponto fundamental do método é medir o componente estrutural. O componente diferencial é meramente um resíduo que, embora possa refletir a ação de inúmeros fatores, pode servir como um guia potencialmente muito útil desses fatores, já que uma variável muito importante já foi removida, isto é, a composição industrial.

Certamente, para se chegar a uma explicação mais completa dos fatores relevantes dos efeitos composição (proporcional) e local (diferencial) são necessários estudos complementares. Nas palavras de Knudsen (2000: 192), embora a análise shift-share não "explique o fenômeno econômico (esta) possibilita alocar variações entre alternativas competitivas (deslocamento setorial nacional versus deslocamento regional, independente do setor), e nos leva mais próximos da explicação”. 
Conforme explicitado anteriormente, apesar de sua simplicidade, o modelo shiftshare tradicional apresenta alguns problemas que os pesquisadores têm discutido e tentado solucionar através de um crescente número de extensões e adições ao modelo. ${ }^{5}$ As mais conhecidas deficiências consideradas na literatura dizem respeito ao fato de que o modelo não apresenta conteúdo teórico ou capacidade de previsão, nem considera as possíveis interações entre os diferentes componentes, especialmente entre a composição industrial e o efeito competitividade. Mesmo sendo considerada uma ferramenta de análise territorial, esta nunca leva em consideração qualquer hipótese acerca das possíveis ligações entre as entidades territoriais. Além disso, os resultados da análise shift-share apresentam grande sensibilidade ao nível de classificação das atividades econômicas utilizado para distinguir os diferentes limites setoriais. Níveis de desagregação mais refinados tendem a elevar a magnitude do componente estrutural e reduzir o efeito competitivo, embora não seja possível estimar a variação entre esses valores.

Cabe salientar, no entanto, que nenhum nível de desagregação pode ser teoricamente justificado. Outro problema salientado na literatura é que essa técnica não leva em conta as mudanças na composição setorial da região estudada durante o período de observação.

Como destacam Danson et al. (1980: 300), muitas das críticas ao modelo shift-share "estão baseadas em uma tendência dos pesquisadores exigirem muito dessa técnica”.

A primeira grande reformulação do modelo shift-share clássico foi dirigida para o problema da interdependência entre os componentes setorial e regional, que os críticos consideravam ser o maior obstáculo enfrentado pelo mesmo (Stevens \& Moore, 1980), dado que ambos os efeitos dependem da estrutura industrial. Esteban-Marquillas (1972) revisou o efeito competitivo da equação clássica introduzindo os conceitos de emprego homotético e efeito alocação. Levando a seguinte identidade shift-share: 


$$
\Delta E=E N_{i j}+E S_{i j}+E C_{i j}^{*}+E A_{i j}
$$

Onde, Efeito Nacional

$$
E N_{i j}=E_{i j} r_{n}
$$

Efeito Setorial ou Estrutural

$$
E S_{i j}=E_{i j}\left(r_{i n}-r_{n}\right)
$$

Efeito Competitivo ou Regional

$$
E S_{i j}=E_{i j}\left(r_{i n}-r_{n}\right)
$$

Efeito Alocação

$$
E A=\left(E_{i j}-E_{i j}^{*}\right)\left(r_{i j}-r_{i n}\right)
$$

O emprego homotético $\left(E_{\dot{j}}^{*}\right)$ é incorporado no efeito competitivo $\left(E^{*}\right)$ para livrá-lo da "influência estrutural regional" e então encerrar sua interdependência com o efeito setorial. O emprego homotético é definido como a magnitude que assumiria o emprego no setor $i$ na região $J$, caso fosse admitido idêntica estrutura industrial na região e na nação (Esteban-Marquillas 1972: 251). Dessa forma, o emprego homotético do setor ${ }^{i}$ na região ${ }^{j}$ é dado pela expressão:

$$
E_{i J}^{*}=\frac{E_{R j}^{0} * E_{i n}^{0}}{E_{n}}
$$

Onde, $E_{R j}=$ emprego em todos os setores ou indústrias na região $j=\Sigma_{i} E_{i j}$; $E_{i n}=$ emprego nacional no setor $i=\Sigma_{j} E_{i j} ; E_{n}=$ emprego total em todos os setores na nação $=\Sigma_{i} \Sigma_{j} E_{i j}$.

Substituindo $E_{i j}^{*}$ por $E_{i j}$, o efeito competitivo se torna:

$$
E C_{i j}^{*}=E_{i j}^{*}\left(r_{i j}-r_{i n}\right)
$$


Conforme se observa, a equação (12) é exatamente igual a equação (9) da nova identidade shift-share elaborada por Esteban-Marquillas (1972), que representa o mesmo efeito competitivo do modelo clássico - equação (4), livre da influência estrutural regional.

O efeito competitivo homotético, $E C_{i j}^{*}$, fornece uma medida da vantagem ou desvantagem comparativa de uma região em comparação com a nação no setor $i$. Para completar a identidade, um novo componente é introduzido - o efeito alocação $\left(E A_{i j}\right)$. Então, o efeito competitivo clássico é decomposto em duas partes: efeito competitivo hemoteticamente ajustado e efeito alocação.

O efeito alocação, por sua vez, é constituído do emprego esperado e do diferencial. O emprego esperado $\left(E_{i j}-E_{j}^{*}\right)$, é uma medida da especialização regional no setor $i$. Este indica se existem mais ou menos pessoas empregadas na indústria $i$ do que seria esperado, considerando o nível médio nacional para aquele setor. Já o segundo elemento do efeito alocação, o diferencial $\left(\left(r_{i j}-r_{i}\right)\right)$, é uma medida da vantagem comparativa regional na indústria $i$ Este indica se a indústria da região está crescendo mais rapidamente que a "mesma" indústria em outras partes da nação. Juntos, estes dois elementos fornecem uma medida de se a região esta se especializando em setores nos quais esta tem uma vantagem comparativa. Quanto maior o total do efeito alocação, "melhor é a distribuição regional do emprego entre os diferentes setores, de acordo com sua respectiva vantagem" (Esteban-Marquillas 1972: 252).

A contribuição de Esteban-Marquillas (1972), acima descrita, expandindo o modelo shift-share clássico é considerada seminal, "pois as principais revisões do modelo clássico após 1972 têm incluído de alguma forma o conceito de homoteticidade” (Selting \& Loveridge 1992: 14).

\subsection{Base de dados}

Os dados empregados no estudo advêm da Relação Anual de Informações Sociais (RAIS), disponibilizadas pelo Ministério do Trabalho e Emprego (MTE), para os anos 1997, 2002 e 2007, em nível macrorregional.

As informações obtidas foram referentes aos grandes setores de atividades. Inicialmente, a análise foi restrita a 8 grandes setores da economia (extrativismo mineral, indústria de transformação, serviços industriais de utilidade pública, construção civil, comércio, serviços, administração pública e agro- 
pecuária), sendo em seguida desagregada para 25 setores visando um estudo mais detalhado do emprego regional.

\section{Análise das desigualdades regionais}

Esta seção tem como objetivo apresentar a avaliação dos dados referentes à evolução do emprego nas regiões e no país como um todo, no período 19972007, procurando identificar sua decomposição através do uso da análise shift-share na sua forma tradicional e do modelo ampliado com a introdução do conceito de emprego homotético e do efeito alocação.

Na seção 4.1, os dados regionais de emprego, estão desagregados em oito setores de atividades, enquanto na aplicação dos modelos shift-share, objeto da seção 4.2, optamos por desagregar mais detalhadamente os dados do emprego regional, classificando-os em 25 setores de atividades, como apresentado nas estatísticas da RAIS.

\subsection{A evolução do emprego nas regiões e no Brasil}

As Tabelas 1 e 2, apresentadas a seguir, foram elaboradas no sentido de possibilitar a análise da evolução do emprego registrado nas estatísticas da RAIS no período 1997-2007 e em cada um dos quinquênios (1997-2002 e 2002-2007), em nível macrorregional e setorial ${ }^{6}$. Enquanto na Tabela 1, os percentuais revelam a distribuição do emprego nacional de cada setor de atividade, a Tabela 2 mostra a composição estrutural de cada uma das regiões.

Através destas, é possível observar a expansão do emprego formal no País. Em dezembro de 1997, havia no Brasil mais de 24 milhões de trabalhadores formalmente empregados, enquanto no final de 2007 esse número havia aumentado para quase 38 milhões, representando um ingresso líquido superior a 13 milhões de trabalhadores, o que corresponde a uma taxa de crescimento do emprego formal de 4,6\% a. a. (56\% no acumulado do período). Esse aumento do emprego não ocorreu, no entanto, de forma simultânea em todas as regiões e setores da mesma forma. Enquanto as regiões Sudeste e Sul reduziram sua participação no total de empregos gerados, nas demais regiões observou-se o aumento de suas respectivas participações, ganhando, portanto, importância relativa no contexto do País. 
TABELA 1. DISTRIBUIÇÃO DO EMPREGO POR REGIÕES SEGUNDO O SETOR DE ATIVIDADE - 1997-2007 (EM PERCENTAGEM E ABSOLUTO)

\begin{tabular}{|c|c|c|c|c|c|c|}
\hline SETOR/REGIÃO & NORTE & NORDESTE & SUDESTE & SUL & $\begin{array}{l}\text { CENTRO- } \\
\text { OESTE }\end{array}$ & $\begin{array}{c}\text { BRASIL } \\
\text { (abs.) }\end{array}$ \\
\hline \multicolumn{7}{|l|}{1997} \\
\hline Extr. Mineral & 5,69 & 19,82 & 55,70 & 12,69 & 6,09 & 105.830 \\
\hline Ind. Transf. & 2,75 & 11,36 & 58,78 & 23,57 & 3,55 & 4.703 .756 \\
\hline Serv. Ind Ut. Publ. & 3,73 & 20,56 & 54,14 & 15,95 & 5,62 & 332.051 \\
\hline Constr. Civil & 3,08 & 16,71 & 57,49 & 15,99 & 6,73 & 1.162 .045 \\
\hline Comércio & 3,47 & 14,32 & 56,99 & 18,51 & 6,70 & 3.668 .782 \\
\hline Serviços & 3,36 & 15,17 & 59,45 & 15,45 & 6,56 & 7.662 .212 \\
\hline Adm. Pública & 6,66 & 22,86 & 43,80 & 15,10 & 11,58 & 5.441 .855 \\
\hline Agropecuária & 2,04 & 15,50 & 54,51 & 18,32 & 9,58 & 997.892 \\
\hline TOTAL & 3,96 & 16,24 & 54,99 & 17,55 & 7,24 & 24.104 .428 \\
\hline \multicolumn{7}{|l|}{2002} \\
\hline Extr. Mineral & 5,28 & 17,67 & 59,87 & 11,55 & 5,63 & $122.8,6$ \\
\hline Ind. Transf. & 3,39 & 12,31 & 53,00 & 26,74 & 4,56 & 5.356 .159 \\
\hline Serv. Ind Ut. Publ. & 4,90 & 19,89 & 51,08 & 16,76 & 7,38 & 319.068 \\
\hline Constr. Civil & 4,56 & 18,61 & 53,08 & 15,82 & 7,93 & 1.048 .251 \\
\hline Comércio & 4,34 & 14,78 & 53,95 & 18,88 & 8,05 & 5.119 .479 \\
\hline Serviços & 3,28 & 14,60 & 58,86 & 16,03 & 7,23 & 9.378 .566 \\
\hline Adm. Pública & 7,96 & 25,87 & 41,51 & 13,19 & 11,47 & 6.991 .973 \\
\hline Agropecuária & 3,46 & 18,30 & 48,12 & 16,36 & 13,76 & 1.207 .672 \\
\hline TOTAL & 4,67 & 17,25 & 52,11 & 17,79 & 8,18 & 29.544.927 \\
\hline \multicolumn{7}{|l|}{2007} \\
\hline Extr. Mineral & 7,53 & 19,28 & 57,67 & 9,41 & 6,12 & 185.444 \\
\hline Ind. Transf. & 3,64 & 12,77 & 52,97 & 25,56 & 5,06 & 7.082 .167 \\
\hline Serv. Ind Ut. Publ. & 5,84 & 19,02 & 51,32 & 17,64 & 6,18 & 364.667 \\
\hline Constr. Civil & 6,10 & 18,43 & 53,74 & 13,65 & 8,09 & 1.617 .989 \\
\hline Comércio & 4,84 & 15,45 & 53,25 & 18,59 & 7,87 & 6.840 .915 \\
\hline Serviços & 3,54 & 14,59 & 58,36 & 16,34 & 7,17 & 11.935 .782 \\
\hline Adm. Pública & 9,05 & 27,23 & 40,74 & 11,69 & 11,30 & 8.198 .396 \\
\hline Agropecuária & 4,89 & 16,62 & 48,39 & 15,17 & 14,93 & 1.382 .070 \\
\hline TOTAL & 5,20 & 17,46 & 51,94 & 17,29 & 8,11 & 37.607 .430 \\
\hline
\end{tabular}

FONTE: Elaboração própria a partir da RAIS/MTE. 
Em termos setoriais, observa-se que dos oito setores analisados, em apenas cinco houve aumento da participação relativa no total de empregos formais (indústria de transformação, serviços industriais de utilidade pública, construção civil, serviços, administração pública e agropecuária). Somente os setores extrativismo mineral (o,44\%, em 1997; 0,49\% em 2007) e comércio (15,22\%, em 1997; 18,19\%, em 2007) apresentaram aumento de participação relativa; sendo este último o responsável por 95\% do aumento setorial. É importante destacar que este crescimento significativo do comércio ocorreu de forma similar em quase todas as regiões do País.

Ainda na Tabela 2 pode ser vista também a importância do setor público como fonte de emprego para importante parcela da população nas diferentes regiões brasileiras. Os números exibidos mostram claramente a existência de uma relação inversa entre o nível de riqueza da região e o percentual da população absorvido pelo setor. Esse fato se torna mais evidente no caso das regiões Norte e Nordeste. Com referência a esta última, a tendência de absorção da mão de obra pelo setor público é crescente quando comparamos os anos de 1997 - 2002 e 2002 - 2007, embora apresente um ligeiro decréscimo entre 2002 e 2007. No final do período o percentual de absorção setorial era superior a um terço do emprego total da região. No caso da região Norte, a última fronteira agrícola do Brasil, esses números ainda se apresentam mais elevados. Embora apresentem uma evolução positiva na participação, a exemplo da região Nordeste, no final do período a absorção de mão de obra pelo setor apresenta-se, em termos percentuais, um pouco inferior ao início do período. 
TABELA 2. DISTRIBUIÇÃO DO EMPREGO REGIONAL E NACIONAL SEGUNDO O SETOR DE ATIVIDADE - 1997-2007 - (EM PERCENTAGEM E ABSOLUTO)

\begin{tabular}{|c|c|c|c|c|c|c|}
\hline SETOR/REGIÃO & NORTE & NORDESTE & SUDESTE & SUL & $\begin{array}{l}\text { CENTRO } \\
\text {-OESTE } \\
\end{array}$ & BRASIL \\
\hline \multicolumn{7}{|c|}{1997} \\
\hline Extr. Mineral & 0,63 & 0,54 & 0,44 & 0,32 & 0,37 & 0,44 \\
\hline Ind. Transf. & 13,55 & 13,65 & 20,86 & 26,20 & 9,55 & 19,51 \\
\hline Serv. Ind Ut. Publ. & 1,30 & 1,74 & 1,36 & 1,25 & 1,07 & 1,38 \\
\hline Constr. Civil & 3,75 & 4,96 & 5,04 & 4,39 & 4,48 & 4,82 \\
\hline Comércio & 13,35 & 13,42 & 15,77 & 16,05 & 14,08 & 15,22 \\
\hline Serviços & 27,00 & 29,68 & 34,37 & 27,98 & 28,78 & 31,79 \\
\hline Adm. Publica & 37,98 & 31,77 & 17,98 & 19,42 & 36,10 & 22,58 \\
\hline Agropecuária & 2,14 & 3,95 & 4,10 & 4,32 & 5,48 & 4,14 \\
\hline TOTAL (Abs.) & 953.667 & 3.915.451 & 13.254.636 & 4.230.918 & 1.745 .992 & 24.104.428 \\
\hline \multicolumn{7}{|c|}{2002} \\
\hline Extr. Mineral & 0,47 & 0,43 & 0,48 & 0,27 & 0,29 & 0,42 \\
\hline Ind. Transf. & 13,16 & 12,94 & 18,44 & 27,24 & 10,11 & 18,13 \\
\hline Serv. Ind Ut. Publ. & 1,13 & 1,25 & 1,06 & 1,02 & 0,97 & 1,08 \\
\hline Constr. Civil & 3,46 & 3,83 & 3,61 & 3,16 & 3,44 & 3,55 \\
\hline Comércio & 16,10 & 14,85 & 17,94 & 18,39 & 17,04 & 17,33 \\
\hline Serviços & 22,30 & 26,87 & 35,85 & 28,61 & 28,07 & 31,74 \\
\hline Adm. Publica & 40,34 & 35,50 & 18,85 & 17,54 & 33,20 & 23,67 \\
\hline Agropecuária & 3,03 & 4,34 & 3,77 & 3,76 & 6,88 & 4,09 \\
\hline TOTAL (Abs.) & 1.379 .761 & 5.095 .390 & 15.396.672 & 5.256 .600 & 2.416 .504 & 29.544.927 \\
\hline \multicolumn{7}{|c|}{2007} \\
\hline Extr. Mineral & 0,71 & 0,54 & 0,55 & 0,27 & 0,37 & 0,49 \\
\hline Ind. Transf. & 13,20 & 13,77 & 19,21 & 27,84 & 11,74 & 18,83 \\
\hline Serv. Ind Ut. Publ. & 1,09 & 1,06 & 0,96 & 0,99 & 0,74 & 0,97 \\
\hline Constr. Civil & 5,05 & 4,54 & 4,45 & 3,40 & 4,29 & 4,30 \\
\hline Comércio & 16,95 & 16,09 & 18,65 & 19,55 & 17,66 & 18,19 \\
\hline Serviços & 21,59 & 26,52 & 35,66 & 30,00 & 28,05 & 31,74 \\
\hline Adm. Publica & 37,94 & 33,98 & 17,10 & 14,74 & 30,38 & 21,80 \\
\hline Agropecuária & 3,46 & 3,50 & 3,42 & 3,23 & 6,76 & 3,67 \\
\hline TOTAL (Abs.) & 1.954 .641 & 6.567 .837 & 19.532.512 & 6.502 .575 & 3.049 .865 & 37.607 .430 \\
\hline
\end{tabular}

FONTE: Elaboração própria a partir da RAIS/MTE.

A Tabela 3 retrata a taxa de crescimento do emprego por região e setor de atividade no período 1997-2007. Nesta, podemos observar que a taxa de crescimento do emprego no Brasil foi de 4,55\% no período analisado, sendo significativamente menor na primeira etapa (1997-2002) quando foram criados cerca de 5,4 milhões de empregos, equivalente a uma taxa de crescimento anual de 4,15\%, quando comparado ao período seguinte (2002-2007), onde esse número sobe para mais de oito milhões e a taxa anual se eleva para 4,94\%. Com base nestes indicadores preliminares, já é possível diagnosticar que cada um dos quinquênios estudados apresentou um comportamento diferente em relação às taxas de crescimento do emprego face ao ambiente internacional, bem como aos objetivos de política econômica delineados pelo governo federal.

Em termos regionais, observamos que no período 1997-2007 as regiões Norte 
e Centro-Oeste foram aquelas que apresentaram as maiores taxas de crescimento anual do emprego - 7,44\% e 5,74\%, respectivamente. Números esses que se mostraram elevados em razão das mesmas se constituírem na fronteira agrícola do país. Esse fato fica bem mais evidente quando examinamos cada um dos quinquênios isoladamente. No primeiro período - 1997-2002, a taxa de crescimento do setor agropecuário na região Norte ultrapassou a taxa de $15 \%$ ao ano, sendo inclusive superior ao dobro da taxa média anual do crescimento do emprego na região que foi de quase $7,7 \%$ - a maior do país no período em consideração. No caso da região Centro-Oeste, esse mesmo número é um pouco mais modesto, mas mesmo assim quase atinge os 11,7\% ao ano. No segundo período a evolução do setor agropecuário nessas regiões perde um pouco sua força inicial. Novamente os números para a região CentroOeste ficam abaixo dos da região Norte, indicando um início do processo de consolidação do avanço da região, mantendo-se apenas esta última como fronteira agrícola. Em paralelo, observa-se nessas regiões um crescimento bastante acelerado do setor comércio, cuja expansão deve estar relacionada ao dinamismo das atividades agropecuárias e a necessidade de fornecimento de insumos e equipamentos, bem como de bens de consumo aos novos indivíduos que afluem às mesmas, atraídos pelo crescimento acelerado da riqueza.

As dificuldades enfrentadas pelo Brasil, no setor externo, no período 19972002 podem ser detectadas através da comparação da evolução das taxas de crescimento do emprego na indústria de transformação entre este período e o seguinte. Como se sabe, esse setor de atividade concentra os bens cuja produção mais de perto acompanha as flutuações do mercado internacional (Azevedo \& Toneto Júnior, 2001; Sabóia 2001, 2005). A região Sudeste, por concentrar mais da metade dos empregos da indústria de transformação no país (ver Tabela 1) foi a mais penalizada. No período, foi a que apresentou a menor taxa de crescimento médio anual de empregos. Em comparação com o país, a taxa de crescimento médio anual do emprego foi inferior a 70\% da média nacional. E no que diz respeito à indústria de transformação em específico, o desempenho da região foi marcadamente decepcionante - os números regionais ultrapassam ligeiramente os $20 \%$ da média setorial do país.

É importante registrar que essa diferença acentuada nas taxas de crescimento do emprego na indústria de transformação, na região Sudeste, reflete igualmente o efeito das políticas públicas que enfatizam a necessidade de proporcionar uma estrutura industrial mais favorável nas demais regiões do país, as quais vêm sendo colocadas em execução por várias décadas. Certamente, um resultado visível das mesmas está expresso na elevada taxa de crescimento do setor na região Sul no período 1997-2002, quando importantes empresas, sobretudo do setor automobilístico, ali se instalaram. Outro número que reflete esse processo de descentralização industrial é a contínua queda da representatividade da região Sudeste como fonte de emprego na indústria de transformação do país. Em termos percentuais, como mostra a Tabela 1, a região Sudeste, entre 1997 e 2007, decresceu sua participação no setor 
industrial nacional de cerca de 60 para menos de 53\%. Por sua vez, a Tabela 2 mostra que, mesmo com a recuperação setorial apresentada pela região Sudeste, no período 2002-2007, acentuadamente influenciada pela melhoria das condições externas, esta ainda não foi suficientemente forte para que a indústria de transformação retornasse ao mesmo índice de representatividade no total do emprego regional do início do período em estudo. Ao longo dos dez anos analisados, a participação do setor no total do emprego da região Sudeste caiu de 20,86\%, em 1997, para 19,21\%, em 2007.

TABELA 3. TAXA DE CRESCIMENTO DO EMPREGO POR REGIÃO E SETOR DE ATIVIDADE - 1997-2007 - (EM PERCENTAGEM)

\begin{tabular}{|c|c|c|c|c|c|c|}
\hline SETOR/REGIÃO & NORTE & NORDESTE & SUDESTE & SUL & $\begin{array}{c}\text { CENTRO- } \\
\text { OESTE }\end{array}$ & BRASIL \\
\hline \multicolumn{7}{|c|}{$1997-2002$} \\
\hline Extr. Mineral & 1,18 & 0,68 & 4,52 & 1,09 & 1,42 & 3,02 \\
\hline Ind. Transf. & 7,04 & 4,30 & 0,53 & 5,26 & 7,94 & 2,63 \\
\hline Serv. Ind Ut. Publ. & 4,75 & $-1,45$ & $-1,94$ & 0,20 & 4,74 & $-0,79$ \\
\hline Constr. Civil & 5,95 & 0,09 & 3,59 & $-2,24$ & 1,23 & $-2,04$ \\
\hline Comércio & 11,78 & 7,57 & 5,73 & 7,31 & 10,88 & 6,89 \\
\hline Serviços & 3,62 & 3,33 & 3,92 & 4,90 & 6,18 & 4,13 \\
\hline Adm. Publica & 8,97 & 7,78 & 4,02 & 2,31 & 4,94 & 5,14 \\
\hline Agropecuária & 15,44 & 7,40 & 1,33 & 1,57 & 11,69 & 3,89 \\
\hline TOTAL (\%) & 7,67 & 5,41 & 3,04 & 4,44 & 6,72 & 4,15 \\
\hline \multicolumn{7}{|c|}{$2002-2007$} \\
\hline Extr. Mineral & 16,57 & 10,50 & 7,78 & 4,23 & 10,42 & 8,59 \\
\hline Ind. Transf. & 7,28 & 6,52 & 5,74 & 4,80 & 7,95 & 5,75 \\
\hline Serv. Ind Ut. Publ. & 6,39 & 1,79 & 2,81 & 3,77 & $-0,87$ & 2,71 \\
\hline Constr. Civil & 15,63 & 8,85 & 9,34 & 5,89 & 9,50 & 9,07 \\
\hline Comércio & 8,32 & 6,91 & 5,69 & 5,63 & 5,51 & 5,97 \\
\hline Serviços & 6,52 & 4,93 & 4,76 & 5,34 & 4,76 & 4,94 \\
\hline Adm. Publica & 5,91 & 4,29 & 2,85 & 0,77 & 2,92 & 3,23 \\
\hline Agropecuária & 10,08 & 0,77 & 2,85 & 1,20 & 4,42 & 2,73 \\
\hline TOTAL (\%) & 7,21 & 5,21 & 4,87 & 4,35 & 4,77 & 4,94 \\
\hline \multicolumn{7}{|c|}{$1997-2007$} \\
\hline Extr. Mineral & 8,76 & 5,48 & 6,14 & 2,65 & 5,82 & 5,77 \\
\hline Ind. Transf. & 7,16 & 5,40 & 3,10 & 5,03 & 7,94 & 4,18 \\
\hline Serv. Ind Ut. Publ. & 5,57 & 0,16 & 0,40 & 1,96 & 1,90 & 0,94 \\
\hline Constr. Civil & 10,71 & 4,38 & 2,67 & 1,74 & 5,28 & 3,37 \\
\hline Comércio & 10,04 & 7,24 & 5,71 & 6,47 & 8,16 & 6,43 \\
\hline Serviços & 5,06 & 4,13 & 4,34 & 5,12 & 5,47 & 4,53 \\
\hline Adm. Publica & 7,43 & 6,02 & 3,43 & 1,55 & 3,93 & 4,18 \\
\hline Agropecuária & 12,72 & 4,03 & 2,09 & 1,38 & 8,00 & 3,31 \\
\hline TOTAL (\%) & 7,44 & 5,31 & 3,95 & 4,39 & 5,74 & 4,55 \\
\hline
\end{tabular}

FONTE: Elaboração própria a partir da RAIS/MTE.

O crescimento da importância do setor público na absorção de acentuada parcela da população empregada no país no período 1997-2002 em todas as regiões brasileiras, à exceção da região Sul, pode ser explicada pela criação de novos municípios no país no período, bem como pela criação do Estado de Tocantins. Ademais, ressalta-se que este crescimento é mais visível nas regiões menos desenvolvidas, portanto ainda muito dependente da participação do governo federal na dinâmica do emprego. 
No exame do comportamento setorial do emprego no Brasil no período 1997-2007, um último elemento merece destaque neste estudo. Trata-se do comportamento do setor de construção civil. Como é mostrado na Tabela 3, as taxas de crescimento anual do setor passaram de um valor negativo de aproximadamente 2\% no quinquênio 1997-2002 para um valor superior a $9 \%$ no quinquênio seguinte, ficando a taxa anual da década em cerca de $3,4 \%$, superando, inclusive, a taxa de evolução do setor agropecuário. Interessante observar que esse comportamento se estendeu de modo mais ou menos uniforme para todas as regiões do país.

A mudança na gestão da política econômica federal parece ter sido responsável direto por esse comportamento setorial. A ascensão ao poder de um governo com tendências políticas de esquerda, caracterizado pela adoção de ações voltadas para a elevação da renda das classes menos favorecidas, bem como para o incremento dos investimentos em obras públicas, foi o principal fator explicativo para o comportamento diverso do setor de construção civil entre os períodos 1997-2002 e 2002-2007. ${ }^{7}$

A Tabela 4, apresentada a seguir, tem como objetivo sintetizar os principais números do crescimento regional do emprego no Brasil no período 19972007.

7 A capacidade do Estado brasileiro de planejar, coordenar e executar uma política de desconcentração regional é uma discussão que gera controvérsia na literatura econômica sobre o tema. Para Cano (2008), o Brasil apresentou períodos de desconcentração positiva (principalmente nos anos setenta) com notável aumento da diversificação da estrutura produtiva industrial e outros de desconcentração aparente (1990-2005) decorrente de efeito estatístico e não de expressivos aumentos territoriais de produção. 
TABELA 4. O CRESCIMENTO DO EMPREGO NO BRASIL E NAS REGIÕES 1997-2007

\begin{tabular}{|c|c|c|c|c|c|c|}
\hline SETOR/REGIÃO & NORTE & NORDESTE & SUDESTE & SUL & $\begin{array}{l}\text { CENTRO- } \\
\text { OESTE }\end{array}$ & BRASIL \\
\hline \multicolumn{7}{|c|}{$\frac{1}{1997-2002}$} \\
\hline Extr. Mineral & 1,18 & 0,68 & 4,52 & 1,09 & 1,42 & 3,02 \\
\hline Ind. Transf. & 7,04 & 4,30 & 0,53 & 5,26 & 7,94 & 2,63 \\
\hline Serv. Ind Ut. Publ. & 4,75 & $-1,45$ & $-1,94$ & 0,20 & 4,74 & $-0,79$ \\
\hline Constr. Civil & 5,95 & 0,09 & 3,59 & $-2,24$ & 1,23 & $-2,04$ \\
\hline Comércio & 11,78 & 7,57 & 5,73 & 7,31 & 10,88 & 6,89 \\
\hline Serviços & 3,62 & 3,33 & 3,92 & 4,90 & 6,18 & 4,13 \\
\hline Adm. Publica & 8,97 & 7,78 & 4,02 & 2,31 & 4,94 & 5,14 \\
\hline Agropecuária & 15,44 & 7,40 & 1,33 & 1,57 & 11,69 & 3,89 \\
\hline TOTAL (\%) & 7,67 & 5,41 & 3,04 & 4,44 & 6,72 & 4,15 \\
\hline \multicolumn{7}{|c|}{$2002-2007$} \\
\hline Extr. Mineral & 16,57 & 10,50 & 7,78 & 4,23 & 10,42 & 8,59 \\
\hline Ind. Transf. & 7,28 & 6,52 & 5,74 & 4,80 & 7,95 & 5,75 \\
\hline Serv. Ind Ut. Publ. & 6,39 & 1,79 & 2,81 & 3,77 & $-0,87$ & 2,71 \\
\hline Constr. Civil & 15,63 & 8,85 & 9,34 & 5,89 & 9,50 & 9,07 \\
\hline Comércio & 8,32 & 6,91 & 5.69 & 5,63 & 5,51 & 5,97 \\
\hline Servicos & 6,52 & 4,93 & 4,76 & 5,34 & 4,76 & 4,94 \\
\hline Adm. Publica & 5,91 & 4,29 & 2,85 & 0,77 & 2,92 & 3,23 \\
\hline Agropecuária & 10,08 & 0,77 & 2,85 & 1,20 & 4,42 & 2,73 \\
\hline TOTAL $(\%)$ & 7,21 & 5,21 & 4,87 & 4,35 & 4,77 & 4,94 \\
\hline \multicolumn{7}{|c|}{$1997-2007$} \\
\hline Extr. Mineral & 8,76 & 5,48 & 6,14 & 2,65 & 5,82 & 5,77 \\
\hline Ind. Transf. & 7,16 & 5,40 & 3,10 & 5,03 & 7,94 & 4,18 \\
\hline Serv. Ind Ut. Publ. & 5,57 & 0,16 & 0,40 & 1,96 & 1,90 & 0,94 \\
\hline Constr. Civil & 10,71 & 4,38 & 2,67 & 1,74 & 5,28 & 3,37 \\
\hline Comércio & 10,04 & 7,24 & 5,71 & 6,47 & 8,16 & 6,43 \\
\hline Servicos & 5,06 & 4,13 & 4,34 & 5,12 & 5,47 & 4,53 \\
\hline Adm. Publica & 7,43 & 6,02 & 3,43 & 1,55 & 3,93 & 4,18 \\
\hline Agropecuária & 12,72 & 4,03 & 2,09 & 1,38 & 8,00 & 3,31 \\
\hline TOTAL (\%) & 7,44 & 5,31 & 3,95 & 4,39 & 5,74 & 4,55 \\
\hline
\end{tabular}

FONTE: Elaboração própria a partir da RAIS/MTE.

Os números apresentados na Tabela 4 evidenciam a desconcentração regional do emprego. Os números apresentados revelam o crescimento acelerado das regiões de fronteira agrícola do país, com destaque para as regiões Norte e Centro-Oeste.

Um exame adicional das causas dessas variações nas taxas de crescimento regional é possível através de sua decomposição para determinar se o crescimento mais acelerado de algumas regiões foi resultado de sua composição industrial mais favorável ou de alguma outra vantagem diferencial. Essa explicação é possível com o emprego da análise shift-share, que é o objeto da próxima seção. 


\subsection{Decomposição do emprego regional}

A aplicação do modelo shift-share "reformulado" foi realizada, inicialmente, para as cinco grandes regiões que compõem o Brasil, e, posteriormente, para o exame dos setores nos quais as regiões tiveram um melhor ou pior desempenho em comparação com a economia nacional.

Conforme já explicitado na seção 3, a análise shift-share, na versão de Esteban-Marquillas (1972), facilita o entendimento das causas das variações do emprego regional ao eliminar a interdependência entre o efeito setorial (industrial mix) e o efeito competitivo, incorporando, neste último, o conceito de "emprego homotético". Dessa forma, para completar a identidade, o efeito competitivo clássico é decomposto em duas partes: o efeito competitivo homoteticamente ajustado e o efeito alocação.

Os resultados da aplicação do modelo para as cinco grandes regiões do país no período 1997-2007, bem como para cada um dos sub períodos da década estão apresentados na Tabela 5. As colunas indicam a variação real do emprego (em termos absolutos), os efeitos nacional, setorial, competitivo e alocação, e o somatório destes efeitos, representado pela última coluna.

TABELA 5. ANÁLISE SHIFT-SHARE DO CRESCIMENTO DO EMPREGO NAS REGIÕES BRASILEIRAS - 1997-2007

\begin{tabular}{|c|c|c|c|c|c|c|}
\hline \multirow[b]{2}{*}{ REGIÕES } & \multirow{2}{*}{$\begin{array}{c}\text { VARIAÇÃO } \\
\text { REAL DO } \\
\text { EMPREGO } \\
\text { (Abs.) }\end{array}$} & \multicolumn{5}{|c|}{ EFEITOS (\%) } \\
\hline & & NACIONAL & SETORIAL & COMPETITIVO & ALOCAÇÃO & TOTAL \\
\hline \multicolumn{7}{|c|}{$1997-2002$} \\
\hline NORTE & 345.717 & 52,67 & 1,99 & 52,07 & $-6,74$ & 100,00 \\
\hline NORDESTE & 955.500 & 78,24 & 2,00 & 28,91 & $-9,15$ & 100,00 \\
\hline SUDESTE & 1.883 .270 & 134,68 & $-2,80$ & $-31,73$ & $-0,15$ & 100,00 \\
\hline SUL & 847.026 & 95,60 & 0,06 & 1,66 & 2,68 & 100,00 \\
\hline CENTRO-OESTE & 579.467 & 57,65 & 4,37 & 51,93 & $-13,95$ & 100,00 \\
\hline \multicolumn{7}{|c|}{ 2002-2007 } \\
\hline NORTE & 658.044 & 61,30 & $-4,62$ & 48,38 & $-5,06$ & 100,00 \\
\hline NORDESTE & 1.708 .440 & 88,49 & $-3,68$ & 15,46 & $-0,26$ & 100,00 \\
\hline SUDESTE & 4.404 .038 & 106,87 & 2,71 & $-9,30$ & $-0,28$ & 100,00 \\
\hline SUL & 1.426 .916 & 110,66 & 1,04 & $-11,17$ & $-0,53$ & 100,00 \\
\hline CENTRO-OESTE & 726.079 & $\begin{array}{r}99,57 \\
\mathbf{1 9 9}\end{array}$ & -2007 & 13,91 & $-7,87$ & 100,00 \\
\hline NORTE & 1.003 .761 & 53,25 & $-2,13$ & 57,62 & $-8,73$ & 100,00 \\
\hline NORDESTE & 2.663 .940 & 82,37 & $-0,71$ & 24,97 & $-6,63$ & 100,00 \\
\hline SUDESTE & 6.287 .308 & 118,42 & 1,03 & $-19,23$ & $-0,21$ & 100,00 \\
\hline SUL & 2.273 .942 & 104,53 & $-0,39$ & $-4,01$ & $-0,13$ & 100,00 \\
\hline CENTRO-OESTE & 1.305 .546 & 75,10 & $-1,14$ & 40,83 & $-14,79$ & 100,00 \\
\hline
\end{tabular}

FONTE: Elaboração própria a partir da RAIS/MTE. 
À primeira vista, observa-se que o efeito nacional é o responsável pela maior parte da variação do emprego nas regiões. ConseqUentemente, podemos verificar que os resultados do emprego no país são determinados principalmente pelas tendências e decisões tomadas no plano federal. Estas são, talvez, as principais forças condutoras dos resultados do emprego em cada uma das regiões.

Como destaca Stilwell (1963: 63), o componente nacional representa "o montante que o emprego regional teria aumentado se este crescesse exatamente a mesma taxa que o emprego na nação como um todo". Assim, quando a variação real do emprego for menor que o efeito nacional, significa que a região teve um desempenho pior que a observada no país; isto é, a variação relativa líquida é negativa. Examinando os números apresentados na tabela, verificamos que a região Sudeste teve um desempenho pior que a média nacional em todos os períodos analisados, especialmente no primeiro quinquênio - 1997-2002. Além da região Sudeste, a outra região que obteve um desempenho abaixo da média do país foi a região Sul, porém em nível bem menor. Ao contrário das regiões mais industrializadas do país, as regiões de fronteira - Norte e Centro-Oeste - foram as que apresentaram melhor desempenho em comparação com a média nacional.

No exame do efeito setorial, não é surpreendente o fato de que a estrutura industrial teve um impacto positivo para a região Sudeste no período 19972007. Para as demais áreas os valores obtidos são negativos, especialmente nas regiões mais pobres e menos desenvolvidas tecnologicamente. Provavelmente, o fato mais estranho seja o valor negativo do efeito estrutural para a região Sudeste no período 1997-2002. No entanto, dado que esse componente é uma medida da mudança no emprego devido à diferença na taxa de crescimento de um setor específico no país e o crescimento médio da nação, facilmente se encontra uma explicação para o fato. Este é decorrente, fundamentalmente, do desempenho bem abaixo da média nacional de setores como o de construção civil, mecânica, metalúrgica e química nesse período, e que estão fortemente concentrados nessa região.

O componente diferencial, no modelo shift-share ampliado de Esteban-Marquillas (1972), é dividido em dois efeitos: competitivo e alocativo. Embora, nos resultados obtidos tenhamos verificado que somente o primeiro contribuiu significativamente para a explicação da variação total do emprego. $\mathrm{O}$ efeito competitivo é uma medida da variação do emprego em um determinado setor na região e a variação do emprego nesse mesmo setor na área de referência. Uma variação negativa para uma determinada região é uma indicação de que esta apresenta uma desvantagem comparativa em relação à área de referência. Surpreendentemente, este efeito é negativo para a região Sudeste em todos os períodos analisados, especialmente no contexto dos argumentos em favor das economias advindas da concentração industrial, aliado às vantagens da proximidade dos mercados consumidores. Cabe destacar ainda que, embora 
os números obtidos neste estudo para o efeito alocação pareçam inconsistentes com o comportamento econômico racional, com as regiões se especializando em setores nos quais lhes faltam uma vantagem comparativa, resultado semelhante foi obtido por Herzog e Olsen (1977), bem como por Danson et al. (1980).

Feitas as considerações sobre os resultados agregados, é interessante observar em quais setores as regiões tiveram um crescimento mais acelerado ou mais lento que o verificado, setorialmente, no país no período 1997-2007. A Tabela 6 apresenta os resultados setoriais do efeito competitivo homotético para cada um dos 25 setores analisados em cada uma das regiões. Um valor positivo é uma indicação de que a região tem uma vantagem comparativa naquele setor em relação ao país; ao contrário, um valor negativo representa uma desvantagem. De acordo com os números obtidos, a região Sudeste foi aquela que apresentou no período 1997-2007 o pior desempenho em relação ao Brasil em todos os setores, à exceção da indústria extrativa mineral e o setor ensino. Esse fraco desempenho regional em relação ao país fez com que a região Sudeste deixasse de gerar mais de um milhão de empregos, caso seu desempenho nos diversos setores tivesse sido idêntico à média dos mesmos à nível nacional. Os resultados obtidos sugerem que essa região se tornou menos competitiva ao longo do período.

Em contraste, a região Norte foi a que mostrou o melhor desempenho no período analisado, com todos os setores apresentando um crescimento acima da média nacional, à exceção das atividades da área de saúde e madeira e mobiliário. Da mesma forma que a região Norte, a outra área de fronteira agrícola do país - região Centro-Oeste -, também apresentou crescimento no emprego acima das taxas setoriais nacionais em 20 dos 25 setores de atividades considerados neste estudo. 
TABELA 6. RESULTADO DO EFEITO COMPETITIVO HOMOTÉTICO DAS REGIÕES BRASILEIRAS NA GERAÇÃO DE EMPREGOS POR SETOR DE ATIVIDADE $-1997-2007$

\begin{tabular}{lr|r|r|r|r}
\hline \multicolumn{1}{c|}{ SETOR/REGIÃO } & NORTE & NORDESTE & SUDESTE & \multicolumn{1}{c|}{ SUL } & \multicolumn{2}{c}{$\begin{array}{c}\text { CENTRO- } \\
\text { OESTE }\end{array}$} \\
\hline EXTR. MINERAL & 2.358 & -823 & 3.605 & -8.436 & 69 \\
MIN. NÃO MET & 6.248 & 13.109 & -16.343 & -2.919 & 7.653 \\
IND. METALURG. & 29.506 & 30.392 & -39.168 & 27.851 & 37.868 \\
IND, MECANICA & 14.959 & 96.359 & -19.371 & 1.298 & 28.860 \\
ELET. E COMUM. & 2.052 & -817 & -9.740 & 7.006 & -311 \\
MAT. TRANSP. & 22.087 & 79.391 & -32.688 & 36.001 & 26.408 \\
MAD. E MOBIL. & -491 & 3.131 & -2.953 & 2.080 & -3.078 \\
PAPEL E GRAF. & 4.965 & 12.128 & -19.520 & 11.145 & 4.702 \\
BOR. FUMO COURO & 2.007 & 16.034 & -11.379 & 257 & 16.469 \\
IND. QUÍMICA & 20.620 & 23.379 & -46.498 & 28.506 & 61.294 \\
IND. TEXTIL & 7.257 & 11.688 & -67.303 & 37.190 & 29.316 \\
IND. CALÇADOS & 14.185 & 70.862 & -11.142 & -16.347 & 18.666 \\
ALIM. E BEB. & 43.310 & -45.635 & -63.811 & 30.973 & 52.460 \\
SER. UTIL PUB. & 8.148 & -4.435 & -10.433 & 6.800 & 2.607 \\
CONSTR. CIVIL & 62.781 & 26.940 & -58.047 & -41.595 & 23.640 \\
COM. VAREJ. & 85.913 & 64.030 & -214.366 & 5.029 & 87.822 \\
COM. ATACAD. & 20.933 & 22.912 & -31.654 & -807 & -433 \\
INST. FINANC. & 3.949 & -1.207 & -10.197 & 338 & 9.708 \\
ADM. TEC. PROF. & 9.928 & 11.609 & -93.543 & 90.700 & 29.655 \\
TRAN. E COMUM. & 9.496 & -41.267 & -3.673 & 27.832 & 8.030 \\
ALOJ. COMUNIC. & 22.739 & 69.799 & -79.381 & -6.401 & 14.985 \\
MED. ODON. VET. & -12.087 & -27.801 & 28.029 & 18.214 & -767 \\
ENSINO & 5.175 & -34.856 & 657 & 21.441 & 36.017 \\
ADM. PÚBLICA & 116.254 & 253.981 & -315.024 & -325.565 & -14.523 \\
AGRICULTURA & 76.064 & 16.164 & -85.340 & -41.691 & 55.898 \\
TOTAL & 578.355 & 665.067 & -1.209 .283 & -91.102 & 533.014 \\
\hline
\end{tabular}

FONTE: Elaboração própria a partir da RAIS/MTE.

É importante destacar também o desempenho setorial da região Nordeste, que, em termos de empregos homotéticos, foi a que obteve maior vantagem comparativa em relação ao Brasil como um todo. No entanto, deve ser destacado que esse resultado foi fortemente dependente do desempenho no setor de emprego público. Finalmente, cabe ressaltar que a região Sul também teve um desempenho pobre em relação ao crescimento setorial do emprego no Brasil no período 1997-2007. Seu desempenho setorial global ficou abaixo da média nacional.

\section{Considerações Finais}

A geração de emprego em regiões menos desenvolvidas foi superior ao observado em regiões mais desenvolvidas economicamente, e isso traz de volta o debate acerca das causas desse processo. Os efetivos resultados observados da dinâmica do emprego na economia brasileira mostrados neste artigo indicam que está havendo um processo de desconcentração regional. As causas deste processo, todavia, são abordadas na literatura em diferentes visões. Existem os que consideram a recente desconcentração regional como 
resultado de uma política nacional desenvolvimentista, e aqueles que a consideram decorrente basicamente de fatores externos, deixando em dúvida a capacidade do Estado enquanto responsável por coordenar um processo de desenvolvimento regional.

Neste trabalho procuramos analisar a evolução regional e setorial do emprego no Brasil, no período 1997-2007, através da versão de Esteban-Marquillas (1972) que introduziu o conceito de emprego homotético na metodologia tradicional do modelo shift-share sem, contudo, emitir maior juízo de valor em relação às causas dessa evolução, procurando apenas retratar a sua dinâmica.

O modelo shift-share reformulado adotado neste artigo consiste numa técnica de análise "ex-post", que permite decompor a variação do emprego em uma região em quatro componentes aditivos: nacional, estrutural, competitivo e alocativo. Apesar das críticas recebidas, devido às suposições que incorpora, o modelo shift-share se mostra bastante importante no contexto da implantação de políticas públicas, devido ao fato de que os tomadores de decisão quase sempre se veem diante de uma enorme quantidade de informações que precisam ser analisadas rapidamente com o uso de técnicas que não são matematicamente complexas e nem intensivas em dados.

A comparação entre as taxas de crescimento do emprego mostra que existem diferenças significativas entre as diversas regiões e setores do país. Isso é resultado do crescimento contínuo das áreas de "fronteira agrícola" no Brasil - regiões Norte e Centro-Oeste, possibilitando que, em conjunto, criassem mais de um milhão de empregos acima da média nacional. Em contraste, embora a região Sudeste permaneça sendo a que gera mais empregos em números absolutos, em termos relativos apresentou um número inferior de postos de trabalho em comparação com as demais regiões, e isso é fruto, principalmente, das políticas públicas de desenvolvimento regional e industrial que visam à desconcentração industrial no país e que tem sido implantadas por várias décadas.

Na forma que o modelo é especificado, observou-se que a influência da composição estrutural inicial das regiões tem uma pequena influência para explicar as diferenças de variação nas taxas de crescimento relativas do emprego entre as mesmas. Ao contrário, a maior parte do crescimento relativo do emprego é explicada pelo efeito nacional, o que mostra que as decisões de política e os problemas no plano nacional é que são relevantes para a determinação da variação do emprego nas diversas regiões do país. Outro componente importante para explicar a variação regional do emprego no país foi o efeito competitivo, embora sua importância tenha tido uma variação bastante ampla entre as diversas regiões do país.

Por fim, ressalta-se a importância de analisar a evolução do mercado de trabalho regional enquanto variável fundamental para o processo de desenvolvi- 
mento econômico homogêneo, destacando-se o fato da pequena influência do efeito estrutural na geração de emprego regional. Nestes termos, as políticas públicas de emprego devem cada vez mais assumir o seu papel de elemento norteador do desenvolvimento local através, principalmente, de suas políticas ativas que visem dinamizar a economia, incentivando a geração de novos empregos e a manutenção dos empregos já existentes.

\section{Referências}

ALVES, T. W. \& CAMPREGHER, G. A.(2008). Dinâmica do emprego no Brasil de 1995 a 2005. In: XXXVI Encontro Nacional da ANPEC, Anais eletrônicos... Salvador. URL: <http://www.anpec.org.br/encontro2008/artigos/200807191154410-. pdf>, Acesso em: 13 de abril de 2009.

AMBRÓZIO, M. A. G. (2009) Desaceleração do emprego não deve repetir anos 90. Visão do desenvolvimento. BNDES. Nº 62.

ARCELUS, F. J.(1984). “An Extension of Shift-Share Analysis”. Growth and Change. 15(1): 3-8.

ARMSTRONG, H. \& TAYLOR, J. (1985) Regional Economics \& Policy. Oxford: Philip Allan, 1985.

AZEVEDO, P. F.; TONETO JÚNIOR, R. (2001) "Relocalização do emprego industrial formal no Brasil na década de 90”. Pesquisa e Planejamento Econômico, 31(1): 153-186.

BARLOW REPORT. (1940) Royal Commission on the Distribution of the Industrial Population Report. London: HMSO, Command 6153, 1940.

BARROS, R. C. \& SABOIA, J. \& KUBRUSLY, L. S. (2008) "Diferenciação Regional da Geração de Empregos Formais no Brasil no Quadriênio 2003/2006”. Revista Econômica do Nordeste, Fortaleza, 39(2).

BISHOP, K. C. \& SIMPSON, C. E. (1972) “Components of Change Analysis: problems of alternative aproaches to industrial structure. Regional Studies, 6(1): 59-68.

CANO, W. (2008) Desconcentração Produtiva Regional do Brasil: 1970-2005. Campinas: UNESP.

CHEPTEA, A. \& GAULIER, G. \& ZIGNAGO, S. “World Trade Competitiveness: a disaggregated view by shift-share analysis. Paris: Centre D’Études Prospectives et D'Informations Internationales, Working Paper no . 2005-23. URL: <http:// www.cepii.fr/anglaisgraph/workpap/summaries/2005/wpo5-23.3.htm/> . Acesso em 15 de 04 de 2009.

CREAMER, D.(1980) "Shifts of Manufaturing Industries". In Industrial Location and National Resources, US Govt. Printing Office.

DANSON, M. W. \& LEVER, W.F \& MALCOLM, J. F. (1980) "The Inner City Employment Problem in Great Britain, 1952-76: a shift-share approach, Urban Studies 17: 193-210.

DINC, M.; HAYNES, K. (2005) "Productivity, International Trade and Reference Area Interactions in Shift-Share Analysis: Some operational notes". Growth and Change. [S.1.], 36(3): 374-394. 
DUNN, E. S. (1960). “A Statistical and Analytical Technique for Regional Analysis”. Papers and Proceedings of the Regional Science Association 6(1): 98-112.

ESTEBAN-MARQUILLAS J. M Reinterpretation of Shift-Share Analysis. Regional Science and Urban Economics. [S.1.], v. 2, n. 3, p. 249-55, 1972.

(2000). Regional Convergence in Europe and the Industry mix: a shift share analysis. Regional Science and Urban Economics 30(3): 353-364.

FERNANDEZ, M. M. \& MENÉDEZ, A. J. L.(2005). "Spatial shift-share analysis: new developments and some findings for the Spanish case". 45th Congress of the European Regional Science Association, 2005. URL: http://www.sre.wu-wien. ac.atersa/ersaconfs/ersa05/papers/659.pdf>. Acesso em 15 de abril de 2009.

HAYNES, K. \& MACHUNDA, Z. B. (1987). Considerations in Extending Shift-Share Analysis: note. Growth and Change, 18 (2): 69-78.

HERZOG, H. W. \& OLSEN, R. J. (1977). "Shift-Share Analysis Revisited: the allocation effect and the stability of regional structure”, Journal of Regional Science, 17 (3): 441-54.

KNUDSEN, D. C.(2000) Shift-Share Analysis: further examination of models for the description of economic change, Socio-Economic Planning Sciences, 34 (1): 177-198.

KOCHANOWSKI, P. \& BARTHOLOMEW, W. \& JORAY, P. (1989). “The Shift-Share Methodology: deficiencies and proposed remedies", Regional Science Perspectives, 19 (1): 65-88.

MERRIFIELD, J. The role of shift-share in Regional Analysis. Regional Science Perspectives, 13 (1): 48-54.

MONTALI, L. (1998). “Trabalho e Família sob a Reestruturação Produtiva”.In XI Encontro Nacional de Estudos Populacionais. ABEP.

NAZARA, S. \& HEWINGS, G. J. D. (2004). "Spatial Structure and Taxonomy of Decomposition in Shift-Share Analysis". Growth and Change, 35 (4): 476-490.

PIMENTEL, E. \& HADDAD, E. (2005). “Brazilian Regional Inequalities Reexamined: a spatial approach”. In CARVALHO, J. \& HERMANNS, K. (Org.) Políticas e Desenvolvimento Regional no Brasil. Fortaleza: Fundação Konrad Adenauer.

RAMOS, L. \& FERREIRA, V. (2004). Geração de empregos e realocação espacial no mercado de trabalho brasileiro: 1992-2002. Rio de Janeiro: IPEA. (Texto para Discussão, 1027).

SABOIA, J. (2001) "Descentralização industrial no Brasil na década de noventa: um processo dinâmico e diferenciado regionalmente”. Nova Economia, 11(2): 85-121.

. (2005) Geração de emprego industrial nas capitais e interior do Brasil. Brasília, DF: Senai.

SELTING, A. C.; LOVERIDGE, S. (1992). “A Summary of the Literature on ShiftShare Analysis”. St. Paul: Departament of Agricultural and Applied Economics, University of Minnesota. URL: <http://purl.umn.edu/14086>. Acesso 23 de fevereiro de 2009.

SINGER, P. (1998). Globalização e Desemprego: diagnóstico e alternativas. São Paulo: Contexto, 1998. 
STEVENS, B. H. \& MOORE, C. L. (1980). "A critical Review of the Literature on Shift-Share as a Forecasting Technique", Journal of Regional Science, 20 (4): 419-38.

STILWELL, F. J. B. (1969). "Regional Growth and Structural Adaptation". Urban Studies, 6 (2): 162-178.

TAFNER, P. (2006). Brasil: O estado de uma nação. Mercado de Trabalho, Emprego e Informalidade. Rio de Janeiro: IPEA.

Recebido em: 08 de setembro de 2010

Primeira resposta em: 16 de novembro de 2010

Aceite em: 09 de dezembro de 2010 